indicate its presence when the heart's action is much increased by mental or bodily exertion; or, again, it may be so extensive as to render prolonged existence impossible. Then, also, defective states of the auriculo-ventricular valves are of more serious importance-more immediately prejudicial to lifethan such states of the semilunar valves. Defective circulation, for instance, resulting from diseased aortic valves, may be compensated for by hypertrophy of the left ventricle; and, under such circumstances, so long at least as the mitral valves remain sound, life may be preserved. But when the mitral valves are seriously defective, then congestions, etc., of the lungs, which are its most immediate and necessary consequence, arise, and so render life very precarious.

What are usually known as symptoms of heart disease, are in reality symptoms of the general disorders which result from the obstruction of the circulation here spoken of. These disorders, indeed, often indicate to us, by their severity, the importance of the valvular disease much more than the auscultating signs do. They are manifested in the heart itself, in the lungs, the brain, the abdominal organs, and generally in the different parts and organs of the body.

Pulmonary Symptoms. The intimate anatomical and functional relations, existing between the lungs and the heart, explain why the effects of an impeded circulation of blood through the left side of the heart should be immediately resented by the lungs. The blood no longer passes freely from these organs, along its wonted channels, into the left auricle of the heart, its passage being obstructed, either by the constricted valvular opening, or by the blood which regurgitates through the defective mitral or defective aortic valves. Thus obstructed in its course, the blood accumulates in the lungs, whose functions are in consequence deranged. Breatlilessness is, in fact, often one of the first symptoms which indicate the presence of heart-disease. Congestions, pneumonia, bronchitis, pulmonary apoplexy, and hæmorrhage, cedema, and emphysema of the lungs, are all of them the more or less frequent im. mediate or secondary results of valvular diseases of the heart; the extent of the particular disorder usually corresponding with the degree and amount of the valvular lesion. These congestions of the lungs are apt to take place very suddenly in heart-diseases; and often also disappear very rapidly, under care and uppropriate treatment. The pulmonary hæmorrhage, the abundant bronchial secretion, the pulmonary œdema, and the pleuritic effusions, which so often accompany these diseases, are all more or less natural efforts of the lungs to free themselves from the congestions of their blood-vessels.

Aldominal Symptoms. The abdominal organs, and the brain, likewise suffer from the consequences of the valvular disease ; indeed, there is no organ or part of the body which may not, in extreme cases, be brought, more or less, under its disturbing influence.

Let us see how this happens. The pulmonary circulation being impeded, as above described, the blood no longer flows freely out of the right side of the heart, into and through the pulmonary artery; consequently, it accumulates in the right ventricle and pulmonary artery, and thus presents an obstacle to the return of the blood from the venous system, through the venæ cavæ, into the heart. From these accumulations of blood, necessarily result congestions of blood, and their consequences, in those organs and parts to which the effects of such an obstacle are immediately communicated. The most important consequences, thence resulting, fall, in the abdomen, upon the liver, kidneys, and intestines. The liver sometimes rapidly attains, under these circumstances, a very large size; it may even be felt extending low down into the abdomen, several inches beneath the edge of the ribs. The congestion of the kidneys may give rise to temporary albuminuria, which disappears when the congestion, which occasioned it, is removed. The stomach and intestines manifest various signs of disorders resulting from impeded circulation; amongst which may be mentioned, vomiting, hæmatemesis, and discharges of serum and blood from the bowels. By such discharges, indeed, the oppressed circulation often finds much relief in these cases.

When the disease of the heart is far advanced, it renders the congested state of these organs a permanent condition, and consequently gives rise to other and secondary affections. The digestion, and therefore also nutrition and absorption, are deranged; and their permanent derangements entail conditions incompatible with the due performance of their functions. Then follow dropsies and effusions into the serous cavities; which, again, cause pressure, and so, by their mechanical effect upon internal organs, tend to destroy life. The nutrition being disturbed, the blood is no longer duly supplied with its proper materials, and defective respiration prevents it from undergoing aeration; so that nct only one, but all, the vital functions, are thus, directly or indirectly, disordered, each in its derangement tending to complicate the other, and to the increase of the general disorder, and so to the destruction of life.

The disturbances of the brain do not, generally, show themselves, until the valvular disease is much advanced, and the circulation much embarrassed, except under the slight and passing forms of headaches and occasional giddiness. Of the more serious symptoms of cerebral disorders, of coma and convulsions, I shall have occasion to speak, more fully, here. after.*

\section{DO LEECHES DIE WHEN APPLIED TO THE SKIN IN CASES OF POISONING BY OXALIC ACID ?}

By Henry Hanks, Esq., Mile End Road.

IN the first of two papers published by me, " On the Injurious Effects of Rumex Acetosa," illustrated by cases observed during my pupilage at the Bath United Hospital and at the Eastern Dispensary of that city, is a paragraph which relates to the action of oxalic acid on leeches, which acid exists in chemical combination in rumex acetosa, in the form of vinocalate of potash. The paragraph runs thus :- " Four leeches were ordered to a part of the body; the skin to which they were to be applied was cleansed; afterwards, they were retained, one at a time, by means of a wineglass. They would not adhere. After being kept in contact with the surface of the hody ten minutes, they all successively died. On the ensuing day, another supply was recommended: these could not be made to perform suction, and they lived. I state this from just having read: 'In two cases, leeches have been killed by the blood drawn by them from persons who were at the time labouring under the effects of this poison (oxalic acid)' (Taylor's Medical Jurisprudence, 2nd edition, p. 103). And, again, Dr. Beck quotes, 'that leeches applied to the stomach were poisoned, and died: this was six hours after the poison had been taken; and, although healthy, and fastening immediately, yet they did not seem to fill; and, on touching one, it felt hard, and immediately fell off, motionless and dead. ... They had drawn scarcely any blood.'

I cite these facts, as they may be of importance to toxicologists; but I abstain from any comment upon them, as incon. trovertible facts constitute a stable basis on which to found reliable criminal evidence, and need no periphrasis to maintain their truth. In my case, the inquiry was not suggested by a former acquaintance with a similar occurrence, as recorded by any author. The fact was noticed first, and the literary scrutiny was instituted afterwards, when arranging my papers for publication.

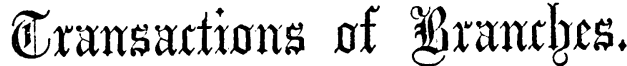

\section{BIRMINGHAM AND MIDLAND COUNTIES BRANCH.}

CASES OF SYPHILITIC DISEASE OF THE CRANIUM.

By James Russell, M.D.

[Read Fcb. 8th, 1860.]

IT is reasonable to suppose that the dura mater, standing, as it does, to the cranial bones in the relation of an internal periosteum, should be liable to suffer from syphilitic disease; and accordingly, experience has shewn, that although such disease may be infrequent in the dura mater, as compared with its occurrence in the membranes strictly called periosteal, yet it does sometimes present itself to our notice, and occasionally in a very obscure form.

Cases of this nature have been described by my late lamented teacher Dr. Todd, by Dr. Graves, and others. Some of these cases are characterised by severe and persistent pain, localised in a particular region of the cranium, sometimes accompanied by tenderness of the scalp, in the painful part. 'This pain may

* I need hardly remind the student, that cardiac dropsies are of a me chanical kind. The position of the fluid varies with the position of the body. Dropsy resulting from renal disease, as is well known, usually shows itself in the first instance in the face and upper parts on the body; ou the other hand, the dropsy of the heart-disea 